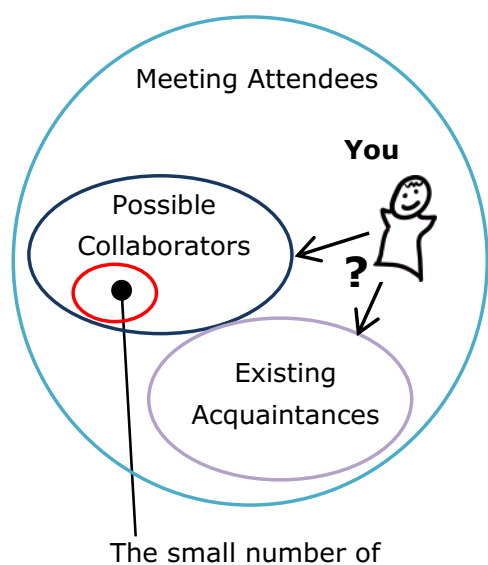

complementary collaborators at

the meeting who fit well with you.

Figure 1: There are two possible paths for you when attending a networking event. Do you a) gravitate towards reconnecting with existing acquaintances? Or b) do you attempt to connect with a new possible collaboration partner? You know you should choose option ' $b$ ', but there are two barriers: Firstly, option ' $a$ ' is the 'comfort zone', and secondly, how can you target possible collaborators when they are hidden amongst all the meeting attendees?

\title{
Well-Connected: Promoting Collaboration by Effective Networking
}

\section{David A. Robb}

Heriot-Watt University

Edinburgh, UK. EH14 4AS

d.a.robb@hw.ac.uk

Thomas S. Methven

Heriot-Watt University

Edinburgh, UK. EH14 4AS

t.methven@hw.ac.uk

\section{Stefano Padilla}

Heriot-Watt University

Edinburgh, UK. EH14 4AS

s.padilla@hw.ac.uk

Paste the appropriate copyright/license statement here. ACM now supports three different publication options:

- ACM copyright: ACM holds the copyright on the work. This is the historical approach.

- License: The author(s) retain copyright, but ACM receives an exclusive publication license.

- Open Access: The author(s) wish to pay for the work to be open access. The additional fee must be paid to ACM.

This text field is large enough to hold the appropriate release statement assuming it is single-spaced in Verdana 7 point font. Please do not change the size of this text box.

Each submission will be assigned a unique DOI string to be included here.

\begin{abstract}
We have developed a method and system that uses mutually agreed problem structuring and self-selection to bring together meeting attendees with complementary interests. The method builds upon previous tools which facilitate structuring and to these we have added method and assistive technology that both facilitates selection, and that records and displays 'connections' in real-time. We have developed these methods over a number of major networking events and discovered that participants both enjoyed this targetted approach and found that it produced more effective collaboration opportunities.
\end{abstract}

\section{Author Keywords}

Interdisciplinary Collaboration; Group Awareness; Visualization; Meeting Tools; Facilitation.

\section{ACM Classification Keywords}

H.5.m. Information interfaces and presentation: Miscellaneous.

\section{Introduction}

Collaboration and innovation are the life blood of science. However, connecting with suitable collaborators whose interests are complimentary to your own is difficult (Figure 1). Existing techniques include exhaustive methods such as speed dating, 


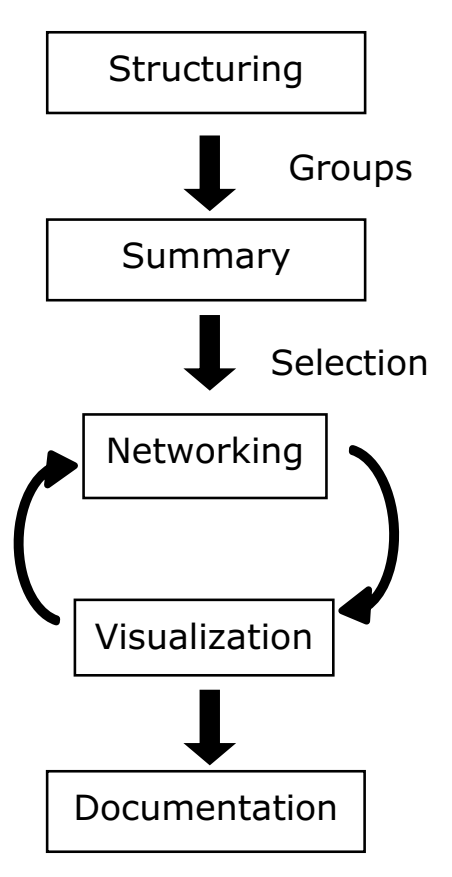

Figure 2: The supported meeting process which leads to effective targeted networking.

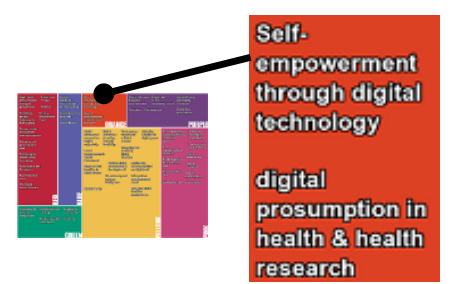

Figure 3: Tree map of ideas submitted and sorted by attendees before a meeting producing colored groups. (One group is enlarged). recommender systems that work off publicly available data, and co-located similarity-based matching $[1,4$, 5]. Speed dating is tiring and rapidly exhausts individuals' attention and retention, while the recommender and matching systems lack the nuanced personal control often required for selecting complimentary and effective partners.

During our work to facilitate research strategy generation we have developed an approach which is designed to bring together researchers with

complementary interests. In the rest of this paper we describe that approach, how it was developed, how it was deployed with a prototype visualization tool (WellConnected) and the initial feedback from users. We conclude with how we will demonstrate this at CSCW.

\section{Our Approach}

Figure 2 outlines the approach detailed below.

Structuring, Leading to Groups

Remotely, prior to a meeting, attendees submit ideas addressing a given problem, then group all the submitted ideas. This is done with an online tool [3] (www.well-sorted.org) producing a structure 'owned' by all attendees e.g. Figure 3. Attendees choose a group to identify with on arrival at the meeting. This results in groups of attendees who share common interests.

\section{Summary, Enabling Selection.}

Group members discuss the ideas in their chosen group and present a 60 second summary (or elevator pitch) of their area to the meeting. Each attendee will have envisaged the interests their ideal complementary collaborator would have. These summaries enable attendees to identify which group(s) to target during networking to find someone with those interests.

\section{Networking}

Attendees are assigned the task of approaching a member of their target group to discuss areas of mutual interest and how they might collaborate. Each round is time limited and attendees note connections.

\section{Visualization}

After each round of networking, details of connections are entered into a visualization (based on the groups from the structuring phase). Projecting this visualization exploits group awareness [2] to further motivate productive networking.

\section{Documentation}

Records of structure, presentations, and networking allow attendees and other interested parties to refer to the meeting's activities afterwards.

\section{Development of the Approach}

The pre-meeting group structuring system [3] was developed and applied over a number of meetings. The networking method and visualization was then developed in three stages described below.

\section{Inter-Group Networking Task}

We introduced a simple task to encourage networking across groups (Figure 4). Facilitators noted that participants were more purposeful than in unstructured sessions and that attendees raised no objection to the idea of making a simple record of the networking.

Visualization Pilot Using Pen and Paper

We took this practice further in a meeting of network leaders. Each attendee undertook several rounds of inter-group networking as described above while keeping a record of each contact on a form which was pre-printed with the meeting structure. Using colored pens, the connections were collated from the forms 

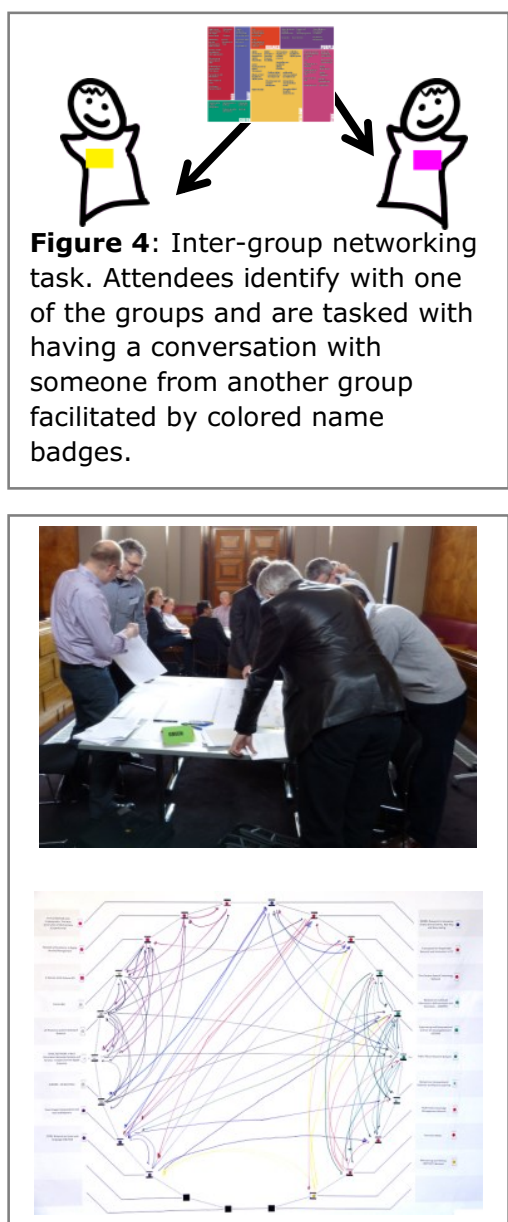

Figure 5: Top: Attendees engaged in the intergroup networking task and noting their connections on pre-printed forms. Bottom: The pilot visualization collated on paper from networking record forms. onto a large (A0) diagram (Figure 5). This was shared with the meeting, stimulating discussion about the interconnectedness of the ideas and scope for collaboration. A major disadvantage of this method, however, was time pressure for facilitators collating the diagram during the meeting. Additionally transcription errors occurred between forms and diagram.

\section{A Visualization Prototype}

To automate the creation of the diagram, a prototype application was built (Figure 6) with these features:

- Layout imported from the meeting structure.

- Minimal data entry effort. Details of the ideas are imported from the meeting structure.

- Connections displayed as lines between ideas with information about the people involved attached.

- Interactivity allows display of: all connections at once, by group, or by individual idea.

- Updates in real time as connections are entered.

- Can be viewed and shared later as a permanent record of the networking which occurred.

\section{Deployment at a structured meeting}

We deployed the prototype networking visualization tool at a funding body sponsored meeting of faculty level researchers from ICT and the humanities. Beforehand, the structure for the meeting was generated by the attendees using the meeting tools. Attendees discussed in their breakout groups and the meeting came together for the summary session to see the 60 -second group presentations. We then gave attendees networking record cards and set the intergroup networking task (Figures 4 and 7 ). The visualization tool (Figure 6) is a web application and, after each networking round, attendees either entered the details of their connection into the system using the iPads and laptops provided, or handed their card to a facilitator. During the session, displayed on a projector, the visualization gradually filled up with connections (Figure 8). An accompanying table which detailed the connections also built up (beneath the visualization). The full visualization was shared at a plenary session which included representatives of funding bodies. The visualization with its connections table formed part of the meeting record along with the pre-meeting structure and the group presentations.

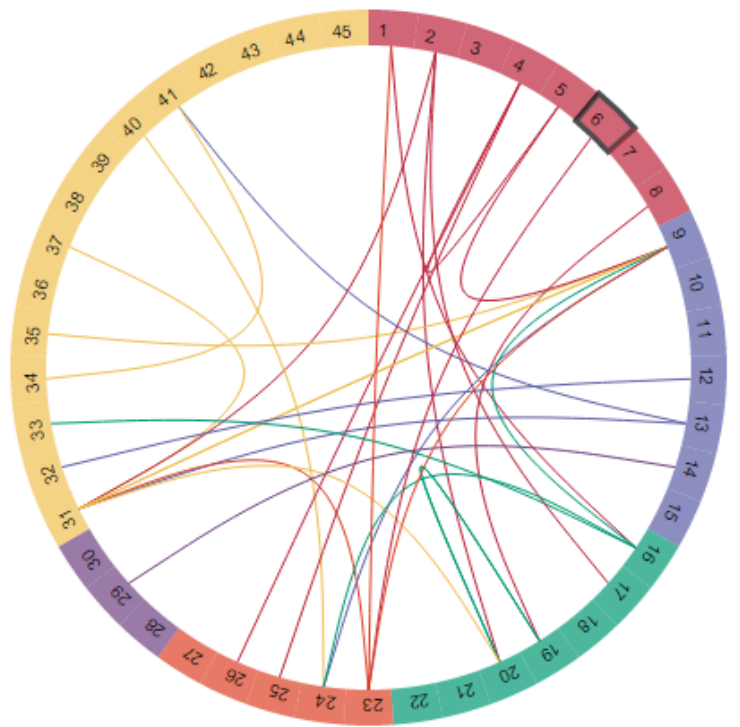

Figure 6: Screen shot from the Well-Connected prototype networking visualization. Numbers around the circumference ink to ideas submitted and sorted into groups pre-meeting by attendees. Colored lines record connections between attendees identifying with the ideas during networking.

Post Meeting Feedback from Attendees

After the meeting we sought feedback from participants using semi-structured, stimulated recall, interviews. The stimuli used were photos of the session and the Well-Connected network visualization. An interesting 

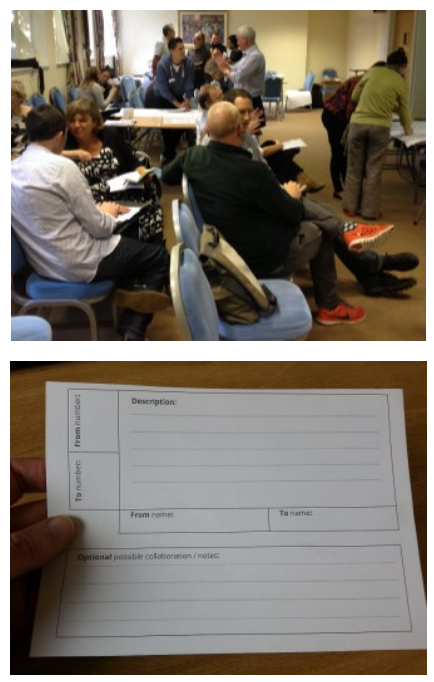

Figure 7. The meeting where the software prototype was deployed Top: Attendees networking with record cards ready to note connection details. Bottom: A blank networking record card.

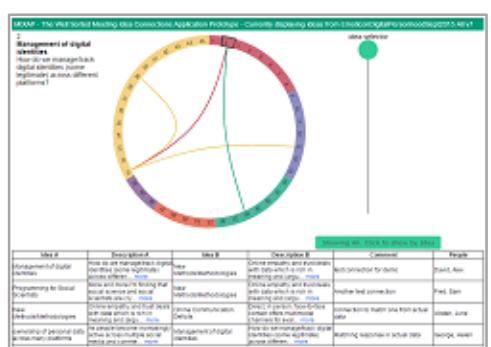

Figure 8. The projected visualization gradually fills up during the networking session. finding was that the networking session had helped a participant connect with a suitable collaborator. They reported they had "collaborated with somebody on a [funding] bid. So it was very successful in that way... because I wouldn't have talked to this person otherwise" [P2]. One theme indicated that group awareness had been achieved for some and this had motivated participants who might not otherwise have got involved to ensure they made connections and entered them into the record: "I think it gives you a more proactive engagement level." And later, "I think you had less opting out...In a less proactive session, you may have a group of people who just chat... they don't get engaged" [P1]. Another theme: "I felt more included... You could see your own ideas in the context of the whole meeting... That visualization.... You see yourself... Are you an outlier? Are you part of that wide picture?... It gives you that sort of [paused]. It's the opposite of sitting there and just consuming. " [P1].

Use of the visualization tool allowed the meeting documentation to include the record of the networking. Looking to the future, participants, when asked, did not object to having their full name noted in the connections record and were willing to have that hyperlinked to their personal URL: "I think it would be a benefit to me post meeting" [P1].

\section{The Well-Connected Demo}

To give CSCW attendees a taste of using WellConnected we will populate the visualization with the CSCW 2016 demo and poster titles and abstracts. Prior to the demo reception, volunteers will sort these for us to produce groups in the same way that the ideas for a meeting would be grouped. At the demo reception attendees will be asked to identify with one of the demo/poster titles as an idea, suggest another that they think shares commonalities, enter this connection using the software, and also interact with the visualization. Attendees will see the accumulation of connections and we hope that authors of the demos/posters at the reception will be motivated to seek out and network with others in the room.

\section{Conclusion}

We have developed and successfully applied an approach in which pre-meeting structuring allows attendees to identify possible complementary collaborators. Visualization can then harness group awareness to further motivate individuals to take part in effective networking and to make those connections that lead to valued collaborations.

\section{Acknowledgements}

We gratefully acknowledge RCUK Digital Economy and EPSRC grants EP/K003542/1 and EP/I038845/1. 'Stick' people drawn by $\mathrm{P}$. Orzechowski.

\section{References}

1. Abouzied, A., \& Chen, J. 2014. CommonTies: a context-aware nudge towards social interaction. In CSCW'14 Companion. 1-4.

2. Kimmerle, J., \& Cress, U. 2008. Group awareness and self-presentation in computer-supported information exchange. IJCSCL, 3(1), 85-97.

3. Methven, T.S., Padilla, S., Corne, D.W., \& Chantler, M.J. 2014. Research strategy generation: avoiding academic 'animal farm'. In CSCW'14 Comp. 25-28.

4. Sousa, G.A.d., Diniz, M.A., Brandao, M.A., \& Moro, M.M. 2015. CNARe: Co-authorship Networks Analysis and Recommendations. In RecSys'15. 329-330.

5. Tang, J., Wu, S., Sun, J., \& Su, H. 2012. Crossdomain collaboration recommendation. In $K D D^{\prime} 12$, 1285-1293. 